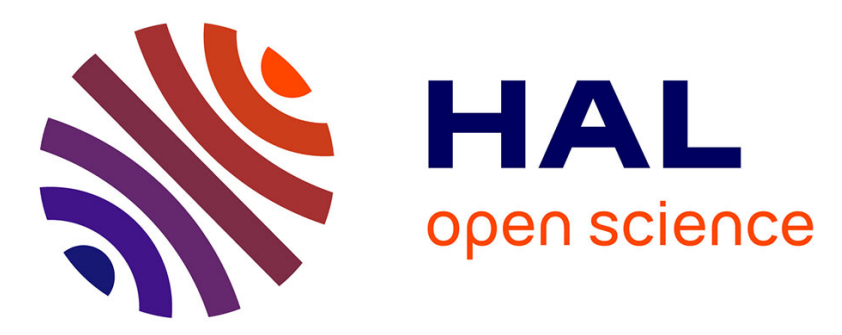

\title{
On the robustness of homogeneous systems and a homogeneous small gain theorem
}

\author{
Emmanuel Bernuau, Denis Efimov, Wilfrid Perruquetti
}

\section{To cite this version:}

Emmanuel Bernuau, Denis Efimov, Wilfrid Perruquetti. On the robustness of homogeneous systems and a homogeneous small gain theorem. IEEE MSC 2014, Oct 2014, Nice/Antibes, France. hal01082174

\section{HAL Id: hal-01082174 \\ https://hal.inria.fr/hal-01082174}

Submitted on 12 Nov 2014

HAL is a multi-disciplinary open access archive for the deposit and dissemination of scientific research documents, whether they are published or not. The documents may come from teaching and research institutions in France or abroad, or from public or private research centers.
L'archive ouverte pluridisciplinaire HAL, est destinée au dépôt et à la diffusion de documents scientifiques de niveau recherche, publiés ou non, émanant des établissements d'enseignement et de recherche français ou étrangers, des laboratoires publics ou privés. 


\title{
On the robustness of homogeneous systems and a homogeneous small gain theorem
}

\author{
Emmanuel Bernuau, Denis Efimov, Wilfrid Perruquetti
}

\begin{abstract}
This paper is devoted to the study of the robustness properties stemming from geometric homogeneity. More precisely, we show that a continuous homogeneous system, which is asymptotically stable without perturbation, is always ISS w.r.t. a perturbation. We characterize the asymptotic gain of such a disturbed system and state a so-called homogeneous small gain theorem based on this estimation.
\end{abstract}

\section{INTRODUCTION}

The problem of robustness and stability analysis with respect to external inputs (like exogenous disturbances or measurement noises) for dynamical systems is at the center of attention of many research works [9], [12], [21], [20], [22], [23]. One of the most popular theories, which can be used for this robustness analysis of nonlinear systems, was originated more than 20 years ago [19] and it is based on the Input-to-State Stability (ISS) property and many related notions. The advantages of ISS theory include a complete list of necessary and sufficient conditions, existence of the Lyapunov method extension, a rich variety of stability concepts adopted for different control and estimation problems.

The main tool to check the ISS property for a nonlinear system consists in a Lyapunov function design. As usual, there is no generic approach to select a Lyapunov function for nonlinear systems. Therefore, computationally tractable approaches for ISS verification for particular classes of nonlinear systems are of great importance, and they are highly demanded in applications.

The homogeneity is an intrinsic property of an object on which the flow of a particular vector field, called Euler vector field, operates as a scaling. This property entails a lot of qualitative results for a homogeneous object, and is of particular interest in view of stability purposes. The notion of homogeneity was found useful by many authors [24], [15], [17], [11], [10], [6], [7], [1].

The ISS properties of homogeneous continuous systems have been studied in [14], [18], [2] and in particular

E. Bernuau is with IRCCyN, UMR CNRS 6597, Ecole Centrale de Nantes, 1 rue de la Noé, 44300 Nantes, France; emmanuel.bernuau@irccyn.ec-nantes.fr.

D. Efimov and W. Perruquetti are with Non-A team of INRIA - LNE, Parc Scientifique de la Haute Borne, 40 avenue Halley, Bat.A, Park Plaza, 59650 Villeneuve d'Ascq, France and with LAGIS UMR CNRS 8219, Ecole Centrale de Lille, BP 48, Cité Scientifique, 59651 Villeneuve-d'Ascq, France; denis.efimov@inria.fr, wilfrid.perruquetti@inria.fr. the links between ISS properties and finite-time stability have been studied in [13]. Recently, in [5], more general robustness results for weighted homogeneous systems have been proven. Our first objective here is to extend the methods of this paper to generalize some results in the more general framework of geometric homogeneity. In the aforementioned paper, an estimation of the asymptotic gain has been given. Our second objective is to improve this estimation by using homogeneous norms. Indeed, we will show that, considering homogeneous norms, the asymptotic gain can be shown to be a linear function. This fact will allow us to state a homogeneous small gain theorem and see how to use it in examples.

The outline of the paper is as follows. Section II is devoted to the introduction of notations, definitions and results that will be used in the sequel. Section III presents the main results: theorem 11 gives conditions based on homogeneity that are sufficient for ensuring ISS of a disturbed system and an estimation of the asymptotic gain; theorem 12 uses this estimation for stating a small gain theorem for homogeneous systems. Finally, a conclusion will sum up the paper and will give some perspectives.

\section{Preliminaries}

\section{A. Notation}

Throughout the paper the following notation is used:

- $\mathbb{R}_{+}=\{x \in \mathbb{R}: x \geq 0\}$, where $\mathbb{R}$ is the set of real number.

- $|\cdot|$ denotes the absolute value in $\mathbb{R},\|$.$\| denotes the$ Euclidean norm on $\mathbb{R}^{n}$.

- if $r_{1}, \ldots, r_{n} \in \mathbb{R}$, we will denote $\operatorname{Diag}\left(r_{1}, \ldots, r_{n}\right)$ the diagonal matrix of dimension $n \times n$ with $k^{\text {th }}$ diagonal entry $r_{k}$.

- For a (Lebesgue) measurable function $d: \mathbb{R}_{+} \rightarrow \mathbb{R}^{m}$, we denote $\|d\|_{[a, b]}=\operatorname{ess}_{\sup } \operatorname{se}_{t a, b]}\|d(t)\|$. If for any $0 \leq a<b$ we have $\|d\|_{[a, b]}<+\infty$, the function $d$ is called locally essentially bounded. We denote $\mathcal{L}_{\text {loc }}^{\infty}$ the set of locally essentially bounded functions $d: \mathbb{R}_{+} \rightarrow \mathbb{R}^{m}$.

- A continuous function $\alpha: \mathbb{R}_{+} \rightarrow \mathbb{R}_{+}$belongs to the class $\mathcal{K}$ if $\alpha(0)=0$ and the function is strictly increasing. A function $\alpha: \mathbb{R}_{+} \rightarrow \mathbb{R}_{+}$belongs to the class $\mathcal{K}_{\infty}$ if $\alpha \in \mathcal{K}$ and it is increasing to infinity. 
- A continuous function $\beta: \mathbb{R}_{+} \times \mathbb{R}_{+} \rightarrow \mathbb{R}_{+}$belongs to the class $\mathcal{K} \mathcal{L}$ if $\beta(\cdot, t) \in \mathcal{K}_{\infty}$ for each fixed $t \in \mathbb{R}_{+}$ and if for each fixed $s \in \mathbb{R}_{+}$the function $t \mapsto \beta(s, t)$ is decreasing to 0 .

- The notation $d_{x} V$ (resp. $\left.d_{x} \Phi\right)$ stands for the differential of the function $V$ (resp. the diffeomorphism $\Phi)$ at the point $x$.

\section{B. Stability notions}

In this paper we will use classical stability notions, recalled hereafter.

Consider the system

$$
\dot{x}=f(x), \quad x \in \mathbb{R}^{n} .
$$

- An equilibrium of the system (1) is a point $x_{0} \in \mathbb{R}^{n}$ such that $f\left(x_{0}\right)=0$.

- A solution of the system (1) is the data of a real interval $I$ and a differentiable function $z: I \rightarrow \mathbb{R}^{n}$ such that $\dot{z}(t)=f(z(t))$ for all $t \in I$. A solution $(I, z)$ of the system (1) is a maximal solution if no solution $(J, w)$ of the system $(1)$ is such that $I \varsubsetneqq J$ and for all $t \in I, w(t)=z(t)$.

- An equilibrium $x_{0}$ of the system (1) is stable if for any neighborhood $U$ of $x_{0}$, there exists a neighborhood $V$ of $x_{0}$ such that for any maximal solution $x(t)$ of (1), if $x(0) \in V$, then $x$ is defined for all $t \geq 0$ and $x(t) \in U$ for all $t \geq 0$.

- An equilibrium $x_{0}$ of the system (1) is locally attractive if there exists a neighborhood $U$ of $x_{0}$ such that for any maximal solution $x(t)$ of $(1)$, if $x(0) \in U$, then $x$ is defined for all $t \geq 0$ and $x(t) \rightarrow x_{0}$ when $t \rightarrow+\infty$. If moreover we can take $U=\mathbb{R}^{n}$, the equilibrium $x_{0}$ is said to be globally attractive.

- An equilibrium of the system (1) is locally (resp. globally) asymptotically stable if it is stable and locally (resp. globally) attractive.

\section{Input-to-State Stability}

Let us consider the following nonlinear system:

$$
\dot{x}=f(x, d),
$$

where $x \in \mathbb{R}^{n}$ is the state, $d \in \mathcal{L}_{\text {loc }}^{\infty}$ is the external input and $f: \mathbb{R}^{n+m} \rightarrow \mathbb{R}^{n}$ is continuous. We will also denote $f_{d}(x)=f(x, d)$ and hence $f_{0}(x)=f(x, 0)$.

Definition 1. [8] The system (2) is called input-to-state stable (ISS), if for any input $d \in \mathcal{L}_{\text {loc }}^{\infty}$ and any $x_{0} \in \mathbb{R}^{n}$ there exist some functions $\beta \in \mathcal{K} \mathcal{L}$ and $\gamma \in \mathcal{K}$ such that for any solution $x(t)$ of the equation (2) we have:

$$
\|x(t)\| \leq \beta(\|x(0)\|, t)+\operatorname{ess}_{\sup _{[0, t]}} \gamma(\|d\|) \quad \forall t \geq 0 .
$$

The function $\gamma$ is called a nonlinear asymptotic gain.

This property has the following Lyapunov function characterization.
Definition 2. A smooth function $V: \mathbb{R}^{n} \rightarrow \mathbb{R}_{+}$is called an ISS Lyapunov function for the system (2) if there exist $\alpha_{1}, \alpha_{2}, \alpha_{3} \in \mathcal{K}_{\infty}$ and $\theta \in \mathcal{K}$ such that for all $x \in \mathbb{R}^{n}$ and all $d \in \mathbb{R}^{m}$ :

$$
\begin{aligned}
& \text { (a) } \quad \alpha_{1}(\|x\|) \leq V(x) \leq \alpha_{2}(\|x\|), \\
& \text { (b) } \quad d_{x} V f(x, d) \leq \theta(\|d\|)-\alpha_{3}(\|x\|) .
\end{aligned}
$$

Note that an ISS Lyapunov function can also satisfy the following equivalent condition for some $\chi \in \mathcal{K}_{\infty}$ :

$$
\|x\|>\chi(\|d\|) \Rightarrow d_{x} V f(x, d) \leq-\alpha_{3}(\|x\|) .
$$

Theorem 3. [8] The system (2) is ISS iff it admits an ISS Lyapunov function.

\section{Homogeneity}

Different notions of homogeneity appear in the litterature. The most common one is the weighted homogeneity, based on a particular choice of the coordinates, while the most generic one is the geometric homogeneity, which is coordinate free. We shall more use in the sequel the framework of geometric homogeneity than the framework of weighted homogeneity, so we will introduce the latter as a particular case of the former.

Definition 4. A vector field $\nu$ on $\mathbb{R}^{n}$ is called an Euler vector field if $\nu$ is of class $C^{1}$, complete (i.e. the maximal integral curves are defined on $\mathbb{R}$ ) and if the origin is a globally asymptotically stable equilibrium of $-\nu$.

Example 1. If we consider a matrix $A \in \mathbb{R}^{n \times n}$ such that $-A$ is Hurwitz, the vector field defined by $\nu(x)=A x$ is an Euler vector field. In particular, if $A=\operatorname{Diag}\left(r_{1}, \ldots, r_{n}\right)$ with $r_{1}, \ldots, r_{n}>0$, the vector field $\nu(x)=A x$ is Euler.

Definition 5. Let $\nu$ be an Euler vector field on $\mathbb{R}^{n}$. We denote $\Phi^{s}(x)$ the value of the flow of $\nu$ at time $s$ with initial condition $x$.

- A function $V: \mathbb{R}^{n} \rightarrow \mathbb{R}$ is $\nu$-homogeneous of degree $\kappa \in \mathbb{R}$ if for all $x \in \mathbb{R}^{n}$ and all $s \in \mathbb{R}$ we have $V\left(\Phi^{s}(x)\right)=e^{\kappa s} V(x)$.

- A vector field $f$ on $\mathbb{R}$ is $\nu$-homogeneous of degree $\kappa \in \mathbb{R}$ if for all $x \in \mathbb{R}^{n}$ and all $s \in \mathbb{R}$ we have $f\left(\Phi^{s}(x)\right)=e^{\kappa s} d_{x} \Phi^{s} f(x)$.

Remark 1. If we have $\nu(x)=A x$, with $-A$ Hurwitz, the flow of $\nu$ verifies $\Phi^{s}(x)=\exp (A s) x$. In particular, if $A=\operatorname{Diag}\left(r_{1}, \ldots, r_{n}\right)$ with $r_{1}, \ldots, r_{n}>0$, we find $\Phi^{s}(x)=\operatorname{Diag}\left(e^{r_{1} s}, \ldots, e^{r_{n} s}\right) x$. The homogeneity defined by such an Euler vector field has been the subject of a lot of works. It is usually referred to as weighted homogeneity, the coefficients $r_{1}, \ldots, r_{n}$ are called the weights and $\mathbf{r}=\left[r_{1}, \ldots, r_{n}\right]$ is called the generalized weight [3]. Let us finally mention that homogeneity with respect to an Euler vector field defined by a generalized weight $\mathbf{r}$ is usually simply referred to as $\mathbf{r}$-homogeneity.

Equivalent conditions for homogeneity exist. Given that we will not need them hereafter, we just refer to 
[16], [6] for a complete exposition of the homogeneity theory.

Theorem 6. [17], [24] Consider $f$ a continuous vector field on $\mathbb{R}^{n}$. Assume moreover that there exists an Euler vector field $\nu$ such that $f$ is $\nu$-homogeneous of degree $\kappa$. Then the following properties are equivalent:

- the origin is a globally asymptotically stable equilibrium of the system (2);

- the system (2) admits a class $C^{1} \nu$-homogeneous Lyapunov function of degree $\mu>\max \{0,-\kappa\}$.

Let us now introduce a tool which will be of great use in the sequel.

Definition 7. Let $\nu$ be an Euler vector field on $\mathbb{R}^{n}$. A $\nu$-homogeneous norm is a function $N: \mathbb{R}^{n} \rightarrow \mathbb{R}$ such that:

1) $N$ is positive definite;

2) $N$ is $\nu$-homogeneous of degree 1 ;

3) $N$ is continuous.

Applying theorem 6 to $f=-\nu$, we find the existence of a class $C^{1}$, positive definite and $\nu$-homogeneous of degree $\mu>0$ function $V$. Hence, taking $N=V^{1 / \mu}$ we get that, for any Euler vector field $\nu$, there exists a $\nu$ homogeneous norm.

When considering weighted homogeneity, homogeneous norms can always be explicited. Indeed, if $\mathbf{r}=$ $\left[r_{1}, \ldots, r_{n}\right]$ is a generalized weight, the function

$$
N(x)=\left(\sum_{i=1}^{n}\left|x_{i}\right|^{\frac{\rho}{r_{i}}}\right)^{\frac{1}{\rho}}, \quad x=\left(x_{1}, \ldots, x_{n}\right)
$$

is a $\mathbf{r}$-homogeneous norm for any $\rho>0$.

It is well-known that all norms are equivalent in a finite-dimensional vector space. This property also holds for homogeneous norms, as proved by the following proposition.

Proposition 8. [6] Suppose $V_{1}$ and $V_{2}$ are continuous real-valued positive definite functions on $\mathbb{R}^{n}$, $\nu$ homogeneous of degrees $l_{1}>0$ and $l_{2}>0$, respectively. Then, for every $x \in \mathbb{R}^{n}$,

$$
M_{-}\left[V_{1}(x)\right]^{\frac{l_{2}}{l_{1}}} \leq V_{2}(x) \leq M_{+}\left[V_{1}(x)\right]^{\frac{l_{2}}{l_{1}}}
$$

with

$$
\begin{aligned}
& M_{-}=\min _{\left\{z: V_{1}(z)=1\right\}} V_{2}(z), \\
& M_{+}=\max _{\left\{z: V_{1}(z)=1\right\}} V_{2}(z) .
\end{aligned}
$$

Considering $f$ a $\nu$-homogeneous vector field of degree $\kappa$ and $V$ a $\nu$-homogeneous function of degree $\mu$, a straightforward computation shows that the function $x \mapsto d_{x} V f(x)$ is $\nu$-homogeneous of degree $\kappa+\mu$. This remark implies a better characterization of the decay rate of the homogeneous Lyapunov function in theorem 6 : indeed, applying proposition 8 with $V_{1}(x)=V(x)$ and
$V_{2}(x)=-d_{x} V f(x)$, we find that there exists a constant $c>0$ such that $d_{x} V f(x) \leq-c V(x)^{\frac{\kappa+\mu}{\mu}}$.

Proposition 8 allows us to compare two $\nu$ homogeneous functions. Sometimes, we also need to compare a $\nu$-homogeneous function to a classical norm.

Lemma 9. [4] Let $V: \mathbb{R}^{n} \rightarrow \mathbb{R}$ be a positive definite $\nu$-homogeneous function of degree $\kappa>0$. There exist $\sigma_{-}$ and $\sigma_{+}$two functions of class $\mathcal{K}$ such that for all $x \in \mathbb{R}^{n}$ :

$$
\sigma_{-}(\|x\|) \leq V(x) \leq \sigma_{+}(\|x\|) .
$$

This lemma incidentally proves that the condition (a) of Definition 2 always holds for a homogeneous Lyapunov function.

\section{Robustness of HOMOGEnEOUS Systems}

The ISS property of weighted homogeneous systems has been investigated in [18] and [14]. In [5], more general conditions have been given for checking the ISS of a homogeneous system. This section aims at improving the results of [5] in two directions: first, the results will be proved in the more general framework of geometric homogeneity; second, we shall prove that one may choose a linear asymptotic gain, provided that the ISS condition of Definition 1 is written with homogeneous norms instead of classical norms.

In this section, we assume that the system (2) verifies an additional homogeneity property.

Assumption 10. There exist an Euler vector field $\nu$ on $\mathbb{R}^{n}$ with flow denoted $\Phi^{s}(x)$, an Euler vector field $\tilde{\nu}$ on $\mathbb{R}^{m}$ with flow denoted $\tilde{\Phi}^{s}(d)$ and $\kappa \in \mathbb{R}$ such that for all $x \in \mathbb{R}^{n}$ and all $d \in \mathbb{R}^{m}$ :

$$
f\left(\Phi^{s}(x), \tilde{\Phi}^{s}(d)\right)=e^{\kappa s} d_{x} \Phi^{s} f(x, d) .
$$

Let us remark that, considering $F(x, d)=$ $(f(x, d), 0)^{T}$, this assumption is equivalent to the $(\nu, \tilde{\nu})$-homogeneity of $F$. Moreover, Assumption 10 implies that the vector field $f_{0}$ is $\nu$-homogeneous of degree $\kappa$. Finally, note that this assumption often holds; for instance, consider $\mathbf{r}$ a generalized weight, $f_{0}$ a r-homogeneous vector field on $\mathbb{R}^{n}$ disturbed by $d \in \mathbb{R}^{n}$ so that the disturbed vector field is $f(x, d)=f_{0}(x+d)$ (the effects of sampling can be modeled in this way). Assumption 10 then obviously holds with $\nu=\tilde{\nu}$.

Theorem 11. Assume that $f: \mathbb{R}^{n} \times \mathbb{R}^{m} \rightarrow \mathbb{R}^{n}$ is continuous and that Assumption 10 holds. Assume also that the origin is a globally asymptotically stable equilibrium of the system $\dot{x}=f_{0}(x)$. Then the system (2) is ISS w.r.t. the input $d$ and for any $\nu$-homogeneous norm $N$ and any $\tilde{\nu}$-homogeneous norm $\tilde{N}$, there exist $C>0$ and $\beta \in \mathcal{K} \mathcal{L}$ such that:

$$
\begin{aligned}
& N(x(t)) \leq \max \{\beta(N(x(0)), t) ; \\
& \left.\quad C \operatorname{essip}_{\tau \in[0, t]} \tilde{N}(d(\tau))\right\} .
\end{aligned}
$$


Proof. This proof follows the same steps as the proof of theorem 6 from [5]. The origin being a globally asymptotically stable equilibrium of the $\nu$-homogeneous system $\dot{x}=f_{0}(x)$, by theorem 6 hereinbefore, there exists a smooth $\nu$-homogeneous Lyapunov function $V$ of degree $\mu>0$. We define $N(x)=V(x)^{1 / \mu}$; the function $N$ is hence a $\nu$-homogeneous norm. Let us denote $\mathbb{S}=\{V=1\}=\{N=1\},-a=\sup _{x \in \mathbb{S}} d_{x} V f_{0}(x)<0$ and $b=\sup _{x \in \mathbb{S}}\left\|d_{x} V\right\|$. We have, for any $x \in \mathbb{R}^{n} \backslash\{0\}$, $x=\Phi^{s}(\tilde{x})$ with $\tilde{x} \in \mathbb{S}$ and $s=\ln N(x)$. Moreover:

$$
\begin{aligned}
d_{x} V f_{d}(x)= & d_{x} V f_{0}(x)+d_{x} V\left(\left(f_{d}-f_{0}\right)(x)\right) \\
= & e^{(\kappa+\mu) s}\left[d_{\tilde{x}} V f_{0}(\tilde{x})\right. \\
& \left.\quad+d_{\tilde{x}} V\left(\left(f_{\tilde{\Phi}^{-s}(d)}-f_{0}\right)(\tilde{x})\right)\right] \\
\leq & V(x)^{\frac{\kappa+\mu}{\mu}}[-a \\
& \left.\quad+b \sup _{z \in \mathbb{S}}\left\|\left(f_{\tilde{\Phi}^{-s}(d)}-f_{0}\right)(z)\right\|\right] .
\end{aligned}
$$

Let $\tilde{N}$ be a $\tilde{\nu}$-homogeneous norm. Since $f$ is continuous, there exists $C>0$ such that $\sup _{z \in \mathbb{S}}\left\|\left(f_{d}-f_{0}\right)(z)\right\|<\frac{a}{2 b}$ whenever $\tilde{N}(d)<1 / C$. Using this fact in the beforehand inequation leads to $d_{x} V f_{d}(x) \leq-\frac{a}{2} V(x)^{\frac{\kappa+\mu}{\mu}}$ whenever $\tilde{N}\left(\tilde{\Phi}^{-s}(d)\right)<1 / C$, i.e. whenever $C \tilde{N}(d)<N(x)=$ $V(x)^{1 / \mu}$. Finally we have whether $V(x) \leq[C \tilde{N}(d)]^{\mu}$ or $d_{x} V f_{d}(x) \leq-\frac{a}{2} V(x)^{\frac{\kappa+\mu}{\mu}}$, which leads to equation (4) with

$$
\beta(r, t)= \begin{cases}r e^{-\frac{a}{2} t} & \kappa=0 \\ \max \left\{\left[r^{-\kappa / \mu}+\frac{a \kappa}{2 \mu} t\right]^{-\mu / \kappa}, 0\right\} & \kappa \neq 0\end{cases}
$$

The general result for any homogeneous norm is obtained by equivalence of homogeneous norms (lemma 8), and finally the ISS property is given by lemma 9 .

The constant $C$ of the equation (4) will be called the homogeneous gain.

Theorem 12 (Homogeneous small gain theorem). Consider a system defined on $\mathbb{R}^{n} \times \mathbb{R}^{m}$ by

$$
\left\{\begin{array}{l}
\dot{x}=f(x, \tilde{x}) \\
\dot{\tilde{x}}=\tilde{f}(x, \tilde{x})
\end{array}, \quad x \in \mathbb{R}^{n}, \tilde{x} \in \mathbb{R}^{m},\right.
$$

with $f$ and $\tilde{f}$ being continuous. Assume that the origin of $\mathbb{R}^{n}$ (resp. $\mathbb{R}^{m}$ ) is a globally asymptotically equilibrium of the system $\dot{x}=f(x, 0)$ (resp. $\dot{\tilde{x}}=\tilde{f}(0, \tilde{x})$ ). Assume moreover that there exist $\nu$ and $\tilde{\nu}$ two Euler vector fields on $\mathbb{R}^{n}$ and $\mathbb{R}^{m}$ respectively, which flows are denoted $\Phi$ and $\tilde{\Phi}$, and $\kappa, \tilde{\kappa} \in \mathbb{R}$ such that

$$
\begin{aligned}
& f\left(\Phi^{s}(x), \tilde{\Phi}^{s}(\tilde{x})\right)=e^{\kappa s} d_{x} \Phi^{s} f(x, \tilde{x}) ; \\
& \tilde{f}\left(\Phi^{s}(x), \tilde{\Phi}^{s}(\tilde{x})\right)=e^{\tilde{\kappa} s} d_{\tilde{x}} \tilde{\Phi}^{s} f(x, \tilde{x}) .
\end{aligned}
$$

Let us denote by $C$ and $\tilde{C}$ the homogeneous gains given by theorem 11. If the small gain condition $C \tilde{C}<1$ holds, then the origin is a globally asymptotically stable equilibrium of the system (6).

Proof. The proof is classical. Consider a couple $(r, \tilde{r}) \in$ $\mathbb{R}_{+} \times \mathbb{R}_{+}$. Let $(x, \tilde{x})$ be a solution of (6) with initial condition $\left(x_{0}, \tilde{x}_{0}\right)$ such that $N\left(x_{0}\right) \leq r$ and $\tilde{N}\left(\tilde{x}_{0}\right) \leq \tilde{r}$. For all $0 \leq t_{0} \leq t$ we have:

$$
\begin{aligned}
& N(x(t)) \leq \beta\left(N\left(x\left(t_{0}\right)\right), t-t_{0}\right)+C \sup _{\tau \in\left[t_{0}, t\right]} \tilde{N}(\tilde{x}(\tau)), \\
& \tilde{N}(\tilde{x}(t)) \leq \tilde{\beta}\left(\tilde{N}\left(\tilde{x}\left(t_{0}\right)\right), t-t_{0}\right)+\tilde{C} \sup _{\tau \in\left[t_{0}, t\right]} N(x(\tau)) .
\end{aligned}
$$

Hence, for any $t_{\max } \geq t_{0}$, if $t \in\left[t_{0}, t_{\max }\right]$ we get:

$$
\begin{aligned}
& N(x(t)) \leq \beta\left(N\left(x\left(t_{0}\right)\right), 0\right)+C \sup _{\tau \in\left[t_{0}, t_{\max }\right]} \tilde{N}(\tilde{x}(\tau)), \\
& \tilde{N}(\tilde{x}(t)) \leq \tilde{\beta}\left(\tilde{N}\left(\tilde{x}\left(t_{0}\right)\right), 0\right)+\tilde{C} \sup _{\tau \in\left[t_{0}, t_{\max }\right]} N(x(\tau)) .
\end{aligned}
$$

which yields

$$
\begin{aligned}
\sup _{\tau \in\left[t_{0}, t_{\max }\right]} N(x(\tau)) & \leq \frac{\beta(r, 0)+C \tilde{\beta}(\tilde{r}, 0)}{1-C \tilde{C}}, \\
\sup _{\tau \in\left[t_{0}, t_{\max }\right]} \tilde{N}(\tilde{x}(\tau)) & \leq \frac{\tilde{\beta}(\tilde{r}, 0)+\tilde{C} \beta(r, 0)}{1-C \tilde{C}} .
\end{aligned}
$$

The right hand members being independant of $t_{\max }$, we find that $N(x(t))$ and $\tilde{N}(\tilde{x}(t))$ are bounded. The stability follows.

Furthermore, let us denote

$$
\begin{aligned}
& S\left(t_{0}\right)=\sup _{t \geq t_{0}} N(x(t)), \\
& \tilde{S}\left(t_{0}\right)=\sup _{t \geq t_{0}} \tilde{N}(\tilde{x}(t)),
\end{aligned}
$$

and $\underset{\tilde{\beta}}{\ell}=\lim _{t_{0} \rightarrow+\infty} S\left(t_{0}\right), \tilde{\ell}=\lim _{t_{0} \rightarrow+\infty} \tilde{S}\left(t_{0}\right)$. Since $\beta$ and $\tilde{\beta}$ are class $\mathcal{K} \mathcal{L}$ functions, for any $\varepsilon>0$, there exists $T$ such that for $t-t_{0} \geq T$

$$
\begin{aligned}
& \beta\left(r, t-t_{0}\right) \leq \varepsilon, \\
& \tilde{\beta}\left(\tilde{r}, t-t_{0}\right) \leq \varepsilon .
\end{aligned}
$$

Thus for all $t \geq t_{0}+T$ we have:

$$
\begin{aligned}
& N(x(t)) \leq \varepsilon+C \tilde{S}\left(t_{0}\right), \\
& \tilde{N}(\tilde{x}(t)) \leq \varepsilon+\tilde{C} S\left(t_{0}\right),
\end{aligned}
$$

then

$$
\begin{aligned}
& S\left(t_{0}+T\right) \leq \varepsilon+C \tilde{S}\left(t_{0}\right), \\
& \tilde{S}\left(t_{0}+T\right) \leq \varepsilon+\tilde{C} S\left(t_{0}\right) .
\end{aligned}
$$

Taking the limit when $t_{0} \rightarrow+\infty$, we get

$$
\begin{aligned}
& \ell \leq \varepsilon \frac{1+C}{1-C \tilde{C}}, \\
& \tilde{\ell} \leq \varepsilon \frac{1+\tilde{C}}{1-C \tilde{C}} .
\end{aligned}
$$

The previous inequality being true for any $\varepsilon>0$, we conclude that $\ell=\tilde{\ell}=0$, that is $(x(t), \tilde{x}(t)) \rightarrow(0,0)$.

Remark 2. Let us emphasize that even though each subsystem has to be homogeneous, the whole system has not. Indeed, when $\kappa \neq \tilde{\kappa}$, the whole system is not homogeneous. This point is interesting because it allows us 
to apply the homogeneous small gain theorem to systems that are not necessarily homogeneous.

Example 2. Consider the following system defined on $\mathbb{R}^{2}$ :

$$
\left\{\begin{array}{l}
\dot{x}=-x^{3}+x y \\
\dot{y}=-y+\alpha x^{2}
\end{array},\right.
$$

where $\alpha$ is a real parameter. This system is not weighted homogeneous. However, taking weights [1,2], we find that the homogeneity condition of theorem 12 holds with $\kappa=2$ and $\tilde{\kappa}=0$. Then, a direct computation shows (see proposition 13) that the homogeneous gain $\tilde{C}$ of the second equation is a class $\mathcal{K}$ function of $|\alpha|$. Hence, no matter what the homogeneous gain $C$ of the first equation is, for $|\alpha|$ small enough, the small gain condition holds and we can apply the homogeneous small gain theorem for getting the asymptotic stability. Finally, there exists $\varepsilon>0$ such that, for all $|\alpha|<\varepsilon$, the origin of this system is globally asymptotically stable.

The method used in the previous example can actually be generalized.

Proposition 13. Consider the following system:

$$
\left\{\begin{array}{l}
\dot{x}=f(x, \alpha \tilde{x}) \\
\dot{\tilde{x}}=\tilde{f}(x, \tilde{x})
\end{array},\right.
$$

where $\alpha \in \mathbb{R}$ is a parameter, and $f$ and $\tilde{f}$ are continuous. We assume that there exist linear Euler vector fields $\nu(x)=A x$ and $\tilde{\nu}(\tilde{x})=\tilde{A} \tilde{x}$ such that for all $x \in \mathbb{R}^{n}$, $\tilde{x} \in \mathbb{R}^{m}$ and all $s \in \mathbb{R}:$

$$
\begin{aligned}
& f(\exp (A s) x, \exp (\tilde{A} s) \tilde{x})=e^{\kappa s} \exp (A s) f(x, \tilde{x}) \\
& \tilde{f}(\exp (A s) x, \exp (\tilde{A} s) \tilde{x})=e^{\tilde{\kappa} s} \exp (\tilde{A} s) \tilde{f}(x, \tilde{x}) .
\end{aligned}
$$

Then, there exists $\varepsilon>0$ such that for all $|\alpha|<\varepsilon$, the origin is a globally asymptotically stable equilibrium of the system (7).

Proof. Following theorem 11 we get:

$$
N(x(t)) \leq \beta(N(x(0)), t)+C \sup _{\tau \in[0, t]} \tilde{N}(\alpha \tilde{x}(\tau)) .
$$

Let us denote $\theta(\alpha)=\sup _{\tilde{N}(\tilde{x})=1} \tilde{N}(\alpha \tilde{x})$. The function $\theta$ is clearly continuous and $\theta(0)=0$. Therefore the function $\sigma(r)=\sup _{|\alpha| \leq r} \theta(\alpha)$ is a class $\mathcal{K}$ function. Now for any $\tilde{x} \neq 0$ there exists $\tilde{x}_{0} \in\{\tilde{N}=1\}$ such that $\exp (\tilde{A} s) \tilde{x}_{0}=$ $\tilde{x}$ with $s=\ln (\tilde{N}(\tilde{x}))$ and:

$$
\begin{aligned}
\tilde{N}(\alpha \tilde{x}) & =\tilde{N}\left(\alpha \exp (\tilde{A} s) \tilde{x}_{0}\right) \\
& =e^{s} \tilde{N}\left(\alpha \tilde{x}_{0}\right) \\
& =\tilde{N}(\tilde{x}) \tilde{N}\left(\alpha \tilde{x}_{0}\right) \\
& \leq \tilde{N}(\tilde{x}) \sigma(|\alpha|) .
\end{aligned}
$$

Then (8) becomes:

$$
N(x(t)) \leq \beta(N(x(0)), t)+C \sigma(|\alpha|) \sup _{\tau \in[0, t]} \tilde{N}(\tilde{x}(\tau)),
$$

and choosing $\varepsilon=\sigma^{-1}(1 / C \tilde{C})$ concludes the proof.

\section{CONCLuSion}

In this paper, we studied disturbed homogeneous systems. We extended the results from [5] to the more general framework of geometric homogeneity. We gave an estimation of the asymptotic gain of such disturbed systems using homogeneous norms and showed that, in doing so, the asymptotic gain can be shown to be a linear function. This fact allowed us to state a homogeneous small gain theorem. This theorem, applicable to a class of nonlinear systems, is formulated in a setting similar to linear systems $(C \tilde{C}<1)$.

Future works include the use of these results to the study of the effects of sampling on homogeneous systems and extensions of these results to discontinuous homogeneous systems.

\section{REFERENCES}

[1] A.Yu. Aleksandrov, A.A. Kosov, and A.V. Platonov. On the asymptotic stability of switched homogeneous systems. Systems \& Control Letters, 61(1):127-133, 2012.

[2] Vincent Andrieu, Laurent Praly, and Alessandro Astolfi. Homogeneous Approximation, Recursive Observer Design, and Output Feedback. SIAM J. Control Optimization, 47(4):1814-1850, 2008.

[3] A. Bacciotti and L. Rosier. Lyapunov Functions and Stability in Control Theory. Springer, Berlin, 2nd edition, 2005.

[4] E. Bernuau, D. Efimov, and W. Perruquetti. Robustness of homogeneous and locally homogeneous differential inclusions. In European Control Conference (Accepted), Strasbourg, France, 2014.

[5] E. Bernuau, A. Polyakov, D. Efimov, and W. Perruquetti. Verification of ISS, iISS and IOSS properties applying weighted homogeneity. Systems \& Control Letters, 62(12):1159-1167, 2013.

[6] S. P. Bhat and D. S. Bernstein. Geometric homogeneity with applications to finite-time stability. Mathematics of Control, Signals and Systems, 17:101-127, 2005.

[7] V.S. Bokharaie, O. Mason, and Mark Verwoerd. D-stability and delay-independent stability of homogeneous cooperative systems. Automatic Control, IEEE Transactions on, $55(12): 2882-2885,2010$.

[8] S.N. Dashkovskiy, D.V. Efimov, and E.D. Sontag. Input to state stability and allied system properties. Automation and Remote Control, 72(8):1579-1614, 2011.

[9] J. Doyle, B. Francis, and A. Tannenbaum. Feedback Control Systems. Mac Millan Publishing Co, 1992.

[10] Lars Grüne. Homogeneous state feedback stabilization of homogeneous systems. SIAM J. Control Optimization, 38(4):1288-1314, 2000.

[11] H. Hermes. Homogeneous feedback controls for homogeneous systems. Systems $\&$ Control Letters, 24:7-11, 1995.

[12] David J. Hill and Peter J. Moylan. Dissipative dynamical systems: basic input-output and state properties. J. Franklin Inst., 309(5):327-357, 1980.

[13] Y. Hong, Z. Jiang, and G. Feng. Finite-time input-to-state stability and applications to finite-time control design. SIAM Journal on Control and Optimization, 48(7):4395-4418, 2010.

[14] Yiguang Hong. $\mathrm{H}_{\infty}$ control, stabilization, and input-output stability of nonlinear systems with homogeneous properties. Automatica, 37(7):819-829, 2001.

[15] Matthias Kawski. Nilpotent lie algebras of vectorfields. $J$. reine angew. Math, 388:1-17, 1988.

[16] Matthias Kawski. Geometric homogeneity and stabilization. In Arthur Krener and David Mayne, editors, Proc. IFAC Nonlinear Control Symposium, pages 164-169, Lake Tahoe, CA, 1995.

[17] L. Rosier. Homogeneous Lyapunov function for homogeneous continuous vector field. Systems \& Control Letters, 19:467473, 1992. 
[18] E.P. Ryan. Universal stabilization of a class of nonlinear systems with homogeneous vector fields. Systems $\&$ Control Letters, 26:177-184, 1995.

[19] Eduardo D. Sontag. Smooth stabilization implies coprime factorization. IEEE Trans. Automat. Control, 34(4):435-443, 1989.

[20] Eduardo D. Sontag. The ISS philosophy as a unifying framework for stability-like behavior. In Nonlinear control in the year 2000, Vol. 2 (Paris), volume 259 of Lecture Notes in Control and Inform. Sci., pages 443-467. Springer, London, 2001.

[21] Arjan van der Schaft. $L_{2}$-gain and passivity techniques in nonlinear control, volume 218 of Lecture Notes in Control and Information Sciences. Springer-Verlag London Ltd., London, 1996.

[22] M. Vidyasagar. Input-output analysis of large-scale interconnected systems, volume 29 of Lecture Notes in Control and Information Sciences. Springer-Verlag, Berlin, 1981. Decomposition, well-posedness and stability.

[23] Jan C. Willems. Dissipative dynamical systems. I. General theory. Arch. Rational Mech. Anal., 45:321-351, 1972.

[24] V.I. Zubov. On ordinary differential equations with generalized homogeneous right-hand sides (in Russian). Izvestiya vuzov, Matematika, 1(2):80-88, 1958. 